\title{
Drug Induced Sleep Endoscopy for Obstructive Sleep Apnea Syndrome: An Initial Experience in a Tertiary Level Centre
}

\author{
Bashu D Parajuli' , Lokendra Mandal' ', Megha Koirala' ', Amit S Bhattarai' ${ }^{1}$, Yogesh Neupane ${ }^{2}$, Anil Shrestha' \\ ${ }^{1}$ Department of Anaesthesiology, Maharajgunj Medical Campus, Tribhuvan University Teaching Hospital, Institute \\ of Medicine, Kathmandu, Nepal, ${ }^{2}$ Department of Otorhinolaryngology, Maharajgunj Medical Campus, Tribhuvan \\ University Teaching Hospital, Institute of Medicine, Kathmandu, Nepal
}

\section{Corresponding author:}

\section{Anil Shrestha, MBBS, MD}

Department of Anaesthesiology, Maharajgunj Medical Campus, Tribhuvan University Teaching Hospital, Institute of Medicine, Kathmandu, Nepal.

Email:aanilsh@hotmail.com

Submitted : November 5, 2019

Accepted : December 7, 2019

\begin{abstract}
Drug induced sleep endoscopy (DISE) is a technique of performing endoscopy of the upper airway after inducing sleep by the use of anaesthetic agents in patients suffering from obstructive sleep apnea syndrome (OSAS). The main purpose of DISE is to detect the obstruction or collapse at one or more location in the upper airway, both for the successful diagnosis and management of OSAS. Here we discuss a case of OSAS who underwent DISE under novel anaesthetic agents midazolam and dexmedetomidine.
\end{abstract}

Keywords: Dexmedetomidine, drug induced sleep endoscopy, obstructive sleep apnea syndrome, propofol

\section{INTRODUCTION}

$\mathrm{O}$ bstructive sleep apnea syndrome (OSAS) is a condition in which upper airway collapse or gets obstructed during sleep time. The condition can be due to enlarged nasal turbinates, enlarged tonsils, soft tissue enlargement at pharynx or larynx, large tongue size, or collapse of airway during sleep at single or multiple levels. ${ }^{1}$ The treatment modality includes weight reduction, exercise of pharyngeal muscles, application of Continuous positive airway pressure (CPAP) or the surgery of the airway at one or multiplesettings.

CPAP is the gold standard therapy for OSAS ${ }^{2}$ but the main problem is compliance with CPAP therapy. Surgical approach is good alternative but the outcome is variable. The outcome can be improved by the upper airway endoscopic study done at the sleep time as it represents the dynamic pathophysiology. ${ }^{3}$ Thus drug induced sleep endoscope (DISE) is performed by the use of the novel anaesthetic agents under monitoring in operating room. The common agents used for DISE are midazolam, propofol or dexmedetomidine. ${ }^{4}$

\section{CASE PRESENTATION}

Twenty six year old male (weight 75kg, BMI $24 \mathrm{~kg} /$ $\mathrm{m}^{2}$ ), otherwise healthy, presented with history of snoring, mouth breathing and few apneic episodes during sleep for one year. All his symptoms were gradually increasing. Upper airway examination was grossly normal. Apnea hypopnea index (AHI) was 72 and lowest oxygen saturation was $75 \%$ in polysomnography.

After adequate fasting on the day of DISE, intravenous cannula was secured, and Ringer's lactate was used as maintenance fluid. ECG, noninvasive blood pressure (NIBP) and pulse oximeter were monitored. Nasal cavity was anaesthetized using $10 \%$ lignocaine spray.To facilitate sedation, lights were dimmed, beeping of monitors was silenced and alarms were turned off, and noise was minimized by educating the staffs prior to the procedure and displaying the warning signs like "DISE, Keep silence" on the doors. A comfortable pillow was kept under the head before sedation. Baseline saturation was $98 \%$, NIBP $140 / 70 \mathrm{mmHg}$ and heart rate $80 /$ minute. After confirmation of 
backup required for anaesthetic management and adequate preparation for DISE, sedation was initiated with IV dexmedetomidine infusion at $1 \mathrm{mcg} / \mathrm{kg}$ for 10 minutes and midazolam $1 \mathrm{mg} \mathrm{IV}$ concurrently. After 5 minutes of infusion, there was onset of snoring. A classical hypopnea and apnea cycle with fall in saturation upto $75 \%$ was seen with no change in ECG, heart rate and blood pressure. Nasal endoscope was inserted through right nostril and the dynamic airway anatomy during the period was recorded. Total duration was 8 minutes. After conclusion, dexmedetomidine infusion was stopped. The patient became conscious after 10 minutes. The patient was discharged home after the discharge criteria were met.

\section{DISCUSSION}

In OSAS there is dynamic obstruction at one or multiple levels during the sleep time. Polysomnography or Overnight Sleep study helps to diagnose and grade OSAS. OSAS is graded according to AHI (Mild 5-15, moderate 15-30 and severe $>30) .5$ Our patient had severe OSAS with $\mathrm{AHI}$ of 72 and lowest oxygen saturation level of $75 \%$.

Patient with OSAS produces loud snoring during sleep and has frequent arousals leading to cognitive dysfunction and day time somnolence. ${ }^{6}$ There can be difficult airway and other multi systemic problems including cardio-respiratory problems, which can be progressive over time. ${ }^{7}$ Our patient had day time somnolence. He snored loudly and had few apneic episodes, which were progressive.

Nasoendoscopic evaluation of upper airway of such patient in the awake state would give anatomical idea of the obstruction however true picture cannot be ensured.Thus sleep endoscopy would be the investigation of choice to figure out the dynamic picture of airway. ${ }^{8}$ These patients easily fall asleep any time of the day if comfortable environment is provided. However the duration of sleep and tolerance of nasal endoscopy is questionable. In our case, we had made our operation room dark, minimized the noise as much as possible including the beep of monitors and had offered a comfortable pillow for the patient.

Natural sleep of the patient can be simulated by using anesthetic drugs and drug induced sleep endoscopy can be done to evaluate the patients airway. An ideal anaesthetic agent for DISE should have rapid onset of action, short duration of action, has amnestic property, can easily be titrated to sedation level and has low residual effect for early discharge. ${ }^{5}$ Anaesthetic agents like midazolam, propofol or dexmedetomidine have the above properties so can be used alone or in combination. Such patients are sensitive with the opioids and inhalational agents and hence not recommended for DISE.

Propofol is the most common inducing agent for DISE however it can cause pharyngeal collapse and central apnea, thus increasing the incidence of hypoxia. ${ }^{9}$ Besides, it can also cause haemodynamic instability at the dose. Midazolam has the property of amnesia, rapid onset of action and minimal or low residual sedative effect and can be used for the sedation of such patient however sole midazolam can lead to respiratory obstruction. ${ }^{10}$ Dexmedetomidine, a centrally acting alpha-2 agonist, has sedative, amnesic, analgesic, sympatholytics and minimal respiratory depressive property. The sleep induced by this drug resembles to that of the natural one. ${ }^{11}$ Its use has been validated in many day care surgeries as anaesthetic adjunct.

We performed the case with midazolam and dexmedetomidine. Patient went to deep sleep after 5 minutes of infusion. There were no features of respiratory depression as minimum saturation was $75 \%$ and respiratory rate was $12-16$ per minute. His blood pressure and heart rate were normal throughout.

There are no severe adverse effects associated with DISE in literature. However in DISE, sleep apnea cycle (SAC) is induced before endoscopy in OSA patients. Airway obstruction may be severe than expected and significant desaturation may occur necessitating advanced airway management. It is hence performed in operation theatre with backup preparation and difficult airway gadgets. ${ }^{5}$ Nasal bleeding due to trauma by endoscope, laryngospasm, pulmonary aspiration, cardiac dysrhythmia, and hypertension may also occur during the procedure. ${ }^{12}$ No adverse events occurred in our patient.

The patient underwent septoplasty with bilateral inferior turbinate reduction with tonsillectomy with modified uvulopalatopharyngoplasty for the definite management on a later date.

\section{CONCLUSION}

A case of OSAS was evaluated with DISE using dexmedetomidine and midazolam smoothly.

\section{ACKNOWLEDGEMENTS}

We would like to acknowledge the contribution of the whole surgery team, anaesthesia team, nursing staff and operation theatre staff involved in the procedure. 


\section{CONFLICT OF INTEREST}

None declared.

\section{REFERENCES}

1. Eichler C, Sommer JU, Stuck BA, Hörmann K, Maurer JT. Does drug-induced sleep endoscopy change the treatment concept of patients with snoring and obstructive sleep apnea? Sleep Breath Schlaf Atm. 2013 Mar;17(1):63-8.

2. Blumen $M$, Bequignon $E$, Chabolle $F$. Druginduced sleep endoscopy: A new gold standard for evaluating OSAS? Part I: Technique. Eur Ann Otorhinolaryngol Head Neck Dis. 2017 Apr;134(2):101-7.

3. Hohenhorst W, Ravesloot MJL, Kezirian EJ, de Vries N. Drug-induced sleep endoscopy in adults with sleep-disordered breathing: Technique and the VOTE Classification system. Oper Tech Otolaryngol-Head Neck Surg. 2012 Mar;23(1):118.

4. Atkins JH, Mandel JE. Drug-induced sleep endoscopy: from obscure technique to diagnostic tool for assessment of obstructive sleep apnea for surgical interventions. CurrOpinAnaesthesiol. 2018 Feb;31(1):120-6.

5. Kuk TS, So E, Karm M-H, Kim J, Chi SI, Kim HJ, et al. Anesthetic management for simultaneous druginduced sleep endoscopy and maxillomandibular advancement in a patient with obstructive sleep apnea. J Dent Anesth Pain Med. 2017 Mar;17(1):716.

6. Spicuzza L, Caruso D, Maria GD. Obstructive sleep apnoea syndrome and its management. TherAdv Chronic Dis. 2015 Sep;6(5):273.

7. Bradley TD, Floras JS. Obstructive sleep apnoea and its cardiovascular consequences. The Lancet. 2009 Jan 3;373(9657):82-93.

8. Vanderveken OM. Drug-induced sleep endoscopy (DISE) for non-CPAP treatment selection in patients with sleep-disordered breathing. Sleep Breath Schlaf Atm. 2013 Mar;17(1):13-4.

9. Cho JS, Soh S, Kim EJ, Cho H, Shin S, Kim HJ, et al. Comparison of three sedation regimens for drug-induced sleep endoscopy. Sleep Breath. 2015 May 1;19(2):711-7.

10. Kotecha B, De Vito A. Drug induced sleep endoscopy: its role in evaluation of the upper airway obstruction and patient selection for surgical and non-surgical treatment. J Thorac Dis. 2018 Jan;10(Suppl 1):S40-7.

11. Yoon B-W, Hong J-M, Hong S-L, Koo S-K, Roh H-J, Cho K-S. A comparison of dexmedetomidine versus propofol during drug-induced sleep endoscopy in sleep apnea patients. The Laryngoscope. 2016 Mar;126(3):763-7.

12. Shallik N. Anesthetic Management for Drug Induced Sleep Endoscopy. Middle East J Anaesthesiol. 2015 Jun 1;23:131-5 